\title{
HREM CHARACTERIZATION OF MAGNETIC THIN FILMS AND MULTILAYERS
}

\author{
David J. Smith* \\ * Center for Solid State Science and Department of Physics and Astronomy, Arizona State \\ University, Tempe, AZ 85287
}

The reduced vertical dimensions of magnetic thin films and multilayers lead to major and often unexpected changes in magnetic properties and behavior. In addition to their intrinsic scientific importance, these novel characteristics have obvious direct relevance to current and projected technological needs. In particular, future generations of magnetic storage devices and magnetic field sensors will rely on spin-dependent scattering in arrays of mesoscopic structures such as spin-valves, giant magnetoresistance (GMR) superlattices, and other spintronic devices that are scaled transversely and vertically on the nanometer scale. Implicit to the successful implementation of this technology is a detailed understanding of materials growth mechanisms at this level. Chemical and crystallographic structure must be correlated with micromagnetic structure and dynamic response before the fundamental limits of device performance can be firmly established. The transmission electron microscope and related techniques will continue to make a major contribution to the success of these ongoing investigations.

In the GMR effect, thin ferromagnetic (FM) layers are separated either by thin metallic spacer layers ("spin-valve" - SV) or thin insulating barriers ("magnetic tunnel junction" - MTJ), and the combination may display changes in resistance that can be as large as $40 \%$ or more at room temperature depending on whether the FM layers are coupled ferromagnetically (i.e., parallel spins) or antiferromagnetically (i.e., anti-parallel spins). The former SV configuration forms the basis for the GMR recording head, which is already in widespread commercial use. The latter MTJ configuration has been adopted in the more recent development of non-volatile magnetic randomaccess memory (MRAM) technology [1,2]. The spin-dependent MTJ has attracted much recent interest, with research especially being directed towards optimizing the tunnel barrier thickness, and maximizing the GMR values. Some post-deposition annealing promotes improved GMR, which has been attributed to the enhanced abruptness of the FM-barrier interface, but a monotonic decrease in GMR values occurs for annealing temperatures above about $300^{\circ} \mathrm{C}$, probably as a result of interlayer diffusion [3]. Atomic-level analysis by electron microscopy of structural and chemical changes induced by varying the growth temperature or by post-deposition annealing should play a major role in elucidating the reasons for the enhanced magnetic properties $[4,5]$. Oxidized aluminum $\left(\mathrm{Al}_{2} \mathrm{O}_{3}\right)$ has been the predominant barrier material but there is continuing interest in alternative oxides such as $\mathrm{HfO}_{2}$ and $\mathrm{CoO}$ [6], as well as barriers of $\mathrm{AlN}$ and $\mathrm{AlON}$ [7], which have potential for band-gap and interface engineering. These materials could offer advantages for device applications as well as allowing additional insights to be obtained into the nature of the tunneling phenomena. For example, there has been ongoing debate about whether some part of the enhanced MR in MTJs results from ballistic transport through nanoscale defects in the barrier, but our recent comparisons with superconducting junctions of similar morphology suggest otherwise [8].

An important factor in the practical utilization of thin magnetic films has been the development of antiferromagnetic (AFM) pinning layers, usually Mn-containing binary alloys or oxides of Co or Ni 
[9]. When cooled through their Néel or ordering temperature, in the presence of a strong magnetic field ( 1-10 kOe), such AFM materials become ordered, and uncompensated spins at the AFM surface then cause "pinning" of the magnetization direction for any immediately adjacent FM layer. Hysteresis loops are also shifted sideways (i.e., exchange-biased) by substantial amounts ( 50-200 Oe). Interfacial roughness and chemical effects are expected to play an important role in layer coupling and magnetization reversal, while further changes in behavior are also likely to occur for both thin films and nanostructures because individual grain size and orientation will have an enhanced influence on the magnetic response. Annealing of Mn-alloy AFMs at elevated temperature $\left(\sim 200-300^{\circ} \mathrm{C}\right)$ is usually required to achieve chemical ordering but significant interdiffusion across the FM/AFM interface then becomes inevitable. It was thus significant that spontaneous chemical ordering was observed in $\mathrm{Mn}_{0.52} \mathrm{Pd}_{0.48}$ films grown by molecular beam epitaxy on body-centeredcubic $\mathrm{Fe}(001)$ films at room temperature 10]. In our recent study of $\mathrm{Ni}_{0.5} \mathrm{Co}_{0.5} \mathrm{O}$, it was found that deposition at below room temperature led to smaller columnar grain diameters and a significant increase in the exchange-bias field [11]. These latter two studies emphasize strongly the value of complementary TEM studies for reaching a better understanding of nanoscale magnetic behavior.

Discontinuous metal/insulator multilayers prepared by sputtering from two separate targets have recently started to attract attention because of several practical advantages compared with "traditional" MTJs which require oxidation treatment and some annealing [12]. The problem of incomplete $\mathrm{Al}$ oxidation and/or the presence of metallic pinholes through the insulator are greatly minimized in these discontinuous layers. Moreover, the multilayers are easily prepared, they are generally very stable both chemically and electrically, and, most importantly, they show high MR sensitivity at low fields [13]. Interesting, long-range magnetic correlations that depend on particle size have also been observed in non-percolated granular films [14]. Lorentz microscopy at low magnification is also useful in understanding these effects [15].

[1] S.S.P. Parkin, et al., J. Appl. Phys. 85 (1999) 5828.

[2] S. Tehrani, et al., J. Appl. Phys. 85 (2000) 5822.

[3] S.S.P. Parkin, et al., Appl. Phys. Lett. 75 (1999) 543.

[4] R.E. Dunin-Borkowski, et al., J. Appl. Phys. 85 (1999) 4815.

[5] M.J. Plisch, et al., Appl. Phys. Lett., 79 (2001) 391.

[6] D.J. Smith, et al., J. Appl. Phys. 83 (1998) 5154.

[7] M.M. Schwickert, et al., J. Appl. Phys. 89 (2001) 6874.

[8] E.P. Price, et al., Appl. Phys. Lett. 80 (2002) 285.

[9] K. Takano and A.E. Berkowitz, J. Magn. Magn. Mater. 200 (1999) 552

[10] R.F.C. Farrow, et al., Appl. Phys. Lett. 80 (2002) 808.

[11] D. Martien, et al., Appl. Phys. Lett. 74 (1999) 1314.

[12] B. Dieny, et al., J. Magn. Magn. Mater. 185 (1998) 283.

[13] S. Sankar, et al., Appl. Phys. Lett. 73 (1998) 535.

[14] S. Sankar, et al., J. Magn. Magn. Mater. 221 (2000) 1.

[15] The author is pleased to acknowledge ongoing collaborations with Prof. Ami Berkowitz and his group at UC San Diego as well as Drs. Robin Farrow and Stuart Parkin at IBM Almaden. 